OPEN ACCESS

Edited by:

Alexandros E. Tsouknidas,

University of Western Macedonia,

Greece

Reviewed by

Natalya Kizilova,

Warsaw University of Technology,

Poland

Yang Liu,

The Hong Kong Polytechnic

University, Hong Kong

*Correspondence:

Pedro Forte

pedromiguel.forte@iscedouro.pt; pedromiguelforte@gmail.com

Specialty section:

This article was submitted to

Biomechanics,

a section of the journal

Frontiers in Bioengineering and

Biotechnology

Received: 21 December 2020

Accepted: 15 February 2021

Published: 11 March 2021

Citation:

Forte P, Morais JE, Barbosa TM and Marinho DA (2021) Assessment of Able-Bodied and Amputee Cyclists' Aerodynamics by Computational

Fluid Dynamics.

Front. Bioeng. Biotechnol. 9:644566.

doi: 10.3389/fbioe.2021.644566

\section{Assessment of Able-Bodied and Amputee Cyclists' Aerodynamics by Computational Fluid Dynamics}

\author{
Pedro Forte ${ }^{1,2,3 *}$, Jorge E. Morais ${ }^{2,3}$, Tiago M. Barbosa ${ }^{2,3}$ and Daniel A. Marinho ${ }^{3,4}$ \\ ${ }^{1}$ Department of Sports, Douro Higher Institute of Educational Sciences, Penafiel, Portugal, ${ }^{2}$ Department of Sports Sciences, \\ Instituto Politécnico de Bragança, Bragança, Portugal, ${ }^{3}$ Research Centre in Sports, Health and Human Development, \\ Covilhã, Portugal, ${ }^{4}$ Department of Sports Sciences, University of Beira Interior, Covilhã, Portugal
}

The aim of this study was to compare the aerodynamics of able-bodied and amputee cyclists by computational fluid dynamics. The cyclists' geometry was obtained by a 3D scanner. Three CAD models were created as able-bodied, transtibial (Tt), and transradial (Tr) amputees. Numerical simulations were conducted up to $13 \mathrm{~m} / \mathrm{s}$ with increments of $1 \mathrm{~m} / \mathrm{s}$ to assess drag force. The drag ranged between 0.36 and $39.25 \mathrm{~N}$ for the ablebodied model, 0.36-43.78 for the Tr model and 0.37-41.39 $\mathrm{N}$ for the Tt model. The pressure drag ranged between 0.20 and $22.94 \mathrm{~N}$ for the normal model, $0.21-28.61$ for the Tr model and 0.23-28.02 N for the Tt model. The viscous drag ranged between 0.16 and 15.31 $\mathrm{N}$ for the normal model, $0.15-15.17$ for the Tr model and 0.14-13.38 $\mathrm{N}$ for the Tt model. The rolling resistance (RR) was higher on the able-bodied $(2.23 \mathrm{~N})$, followed by the $\operatorname{Tr}(2.20 \mathrm{~N})$ and $\operatorname{Tt}(2.17 \mathrm{~N})$ models. As a conclusion, the able-bodied cyclist showed less drag, followed by the Tt and Tr models, respectively. The RR presented higher values in the able-bodied, followed by the Tr and Tt models.

Keywords: cycling, amputee, drag, rolling resistance, CFD

\section{INTRODUCTION}

Cycling is one the most popular time-based sports. Drag force (i.e., aerodynamic resistance) plays an important role in elite cycling performance. Several studies have been carried out over the last decade on the aerodynamics of able-bodied cyclists (Defraeye et al., 2010b; Blocken et al., 2013, Blocken et al., 2018a; Forte et al., 2020a). Cycling is also popular among body-disabled people, but research on this cohort of athletes is rather scarce. Arguably, evidence gathered in able-bodied cyclists has been applied in para-cyclists (Dyer, 2016; Dyer and Disley, 2017). However, it remains to be seen if such approach is accurate. One may argue that, for instance, the aerodynamics of able-bodied cyclists and amputee counterparts might be different.

In able-bodied cyclists, drag accounts to $90 \%$ of total resistive forces, at elite racing speeds (Martin et al., 2006). The second main resistive force in cycling is rolling resistance (RR). Thus, to excel, competitive cyclists, must minimize the resistive forces and enhance the propulsive forces (Martin et al., 1998; Candau et al., 1999). To date, at least two studies modeled amputated cyclist with prosthesis (Dyer, 2014; Childers et al., 2015). However, the main performance determinants, such as, drag contribution to the resistive forces was not assessed. 
In para-cycling the classifications are split-up into five classes, according to their condition (WCi, i.e., $i=1,2,3,4$, or 5 with limitations and or amputations in lower- and upper-limbs) (Tweedy and Vanlandewijck, 2011; Liljedahl et al., 2020). The drag is dependent of the form/shape of the object and the athlete's anthropometrics (Forte et al., 2018a). Thus, an amputee may have a smaller surface drag in comparison to an ablebodied counterpart. However, no study is found comparing the aerodynamics of able-bodied and amputee cyclists [e.g., transtibial (Tt) and/or transradial ( $\mathrm{Tr})]$.

It is possible to assess resistive forces based on analytical procedures, experimental testing (coasting deceleration techniques, wind tunnel testing) and numerical simulations by computer fluid dynamics (Blocken and Toparlar, 2015). The latter one enables the assessment of the total drag force and its components. Total drag force is the sum of viscous drag and pressure drag. Viscous drag is strongly dependent on the form or shape of the body or system (e.g., cyclist plus bicycle, cyclist-bicycle system); whereas, pressure drag is the balance of pressure gradient between the front and back boundaries of the body or system (Schlichting and Gersten, 2016; Forte et al., 2018a). Nevertheless, remains yet elusive what is the partial contribution of each drag component to total drag force in amputees. Moreover, as far as our understanding goes, there is no comparison of the partial contribution of each drag component between amputees and able-bodied cyclists. The gold-standard method to assess drag is the wind tunnel testing. The computational fluid dynamics (CFD) analysis presented good adherence to wind tunnel data (Defraeye et al., 2010b; Blocken and Toparlar, 2015; Forte et al., 2015; Blocken et al., 2018b). Thus, CFD is deemed as a valid and reliable technique to assess drag force. Cycling is an unsteady phenomenon. Nevertheless, steady approach (static analysis) has been shown to present a good agreement with dynamic events (Crouch et al., 2014; Griffith et al., 2019).

Rolling resistance is the product between RR coefficient, mass, and gravity. Cyclists aim to minimize RR by mass reduction (bicycle, cyclist or booth) and the use of high-pressure tires with minimal deformation on the ground (Pugh, 1974). In ablebodied cyclists RR accounts about $10 \%$ of the resistive forces above $5 \mathrm{~m} / \mathrm{s}$ (Blocken and Toparlar, 2015). On asphalt surface, Pugh (1974) reported RR values of $6.9 \mathrm{~N}$, and a RR coefficient of 0.0081. Conversely, Candau et al. (1999), noted RR values between 2.6 and $3.5 \mathrm{~N}$, and the RR coefficient between 0.0035 and 0.0039 . However, no study was found comparing RR between able-bodied and amputee cyclists.

It is possible to assess the resistive forces (drag and RR) contribution by a set of analytical procedures based on the outputs of numerical simulations (Forte et al., 2015). One single study compared the total drag and energy cost by CFD and analytical procedures on able-bodied and amputee cyclists (Forte et al., 2020c). However, no details on pressure, viscous and total drag and RR variations were reported. Moreover, there is also a lack of research assessing the drag variations on amputee cyclists.

The aim of this study was to compare the aerodynamics of able-bodied and amputee cyclists ( $\mathrm{Tt}$ and $\mathrm{Tr}$ ) by CFD and analytical procedures. It was hypothesized that drag is higher in able-bodied cyclist, followed-up by the $\mathrm{Tr}$ and $\mathrm{Tt}$ amputee, respectively.

\section{METHODS}

\section{Participant}

An elite level road cyclist competing at national level was recruited for this research. The bicycle was $5 \mathrm{~kg}$ heavy and the cyclist had $65 \mathrm{~kg}$ of body mass. All procedures were in accordance to the Helsinki Declaration regarding human research and a written informed consent by the volunteered participant was obtained beforehand. The Scientific Committee of the Douro Higher Institute of Educational Sciences approved this research.

\section{Research Design}

Computational fluid dynamics enables the assessment of the aerodynamics under highly controlled conditions. This technique shows a high adherence to data collected in wind tunnel testing (Forte et al., 2015). Knowing drag force and RR, it is possible to determine the partial contribution of viscous and pressure to total drag force, as well as, the contribution of total drag and RR to total resistance forces (Candau et al., 1999). Thus, it is possible to provide a comprehensive comparison of the aerodynamics (viscous drag, pressure drag, total drag, and RR) among able-bodied, $\mathrm{Tr}$, and $\mathrm{Tt}$ models.

\section{Scanning}

The bicycle-cyclist's geometry was collected by a Sense 3D scanner (3D Systems, Inc., Canada) and a commercially available software (Sense, 3D Systems, Inc., Canada). The cyclist was in the upright position on the bicycle (Blocken et al., 2018a). The scans were made with the participant in a static position. The geometry was edited and converted to CAD models on Geomagic Studio (3D Systems, United States) CAD models (Forte et al., 2018a). Three CAD models were created based on the single scanned participant: (Figure 1A) able-bodied (scanned); (Figure 1B) $\mathrm{Tr}$ amputee (edited geometry); (Figure 1C) Tt amputee (edited geometry) (Figure 1).

\section{Boundary Conditions}

The 3D boundaries around the bicycle-cyclist system were set with $7 \mathrm{~m}$ of length, $2.5 \mathrm{~m}$ of width and $2.5 \mathrm{~m}$ of height on Ansys Workbench software (Ansys Fluent 16.0, Ansys Inc., Canonsburg, PA, United States). The grid, with more than 42 million of elements, was created around the geometry placed at $2.5 \mathrm{~m}$ distance of the fluid flow inlet portion (Blocken et al., 2013).

To generate the mesh, the automatic meshing was assigned in the Ansys Fluent 16.0. This option allows creating structured and unstructured meshes with good quality. Creating manually a $3 \mathrm{D}$ mesh with similar or even better quality is far more time-consuming and a very convoluted procedure for complex geometries (Marinho et al., 2010). The meshed quality was controlled based on the skewness, orthogonal quality, amount of elements, and Y+ wall turbulence values (Peters, 2009). Three different meshes were made: the polyhedral meshing; 

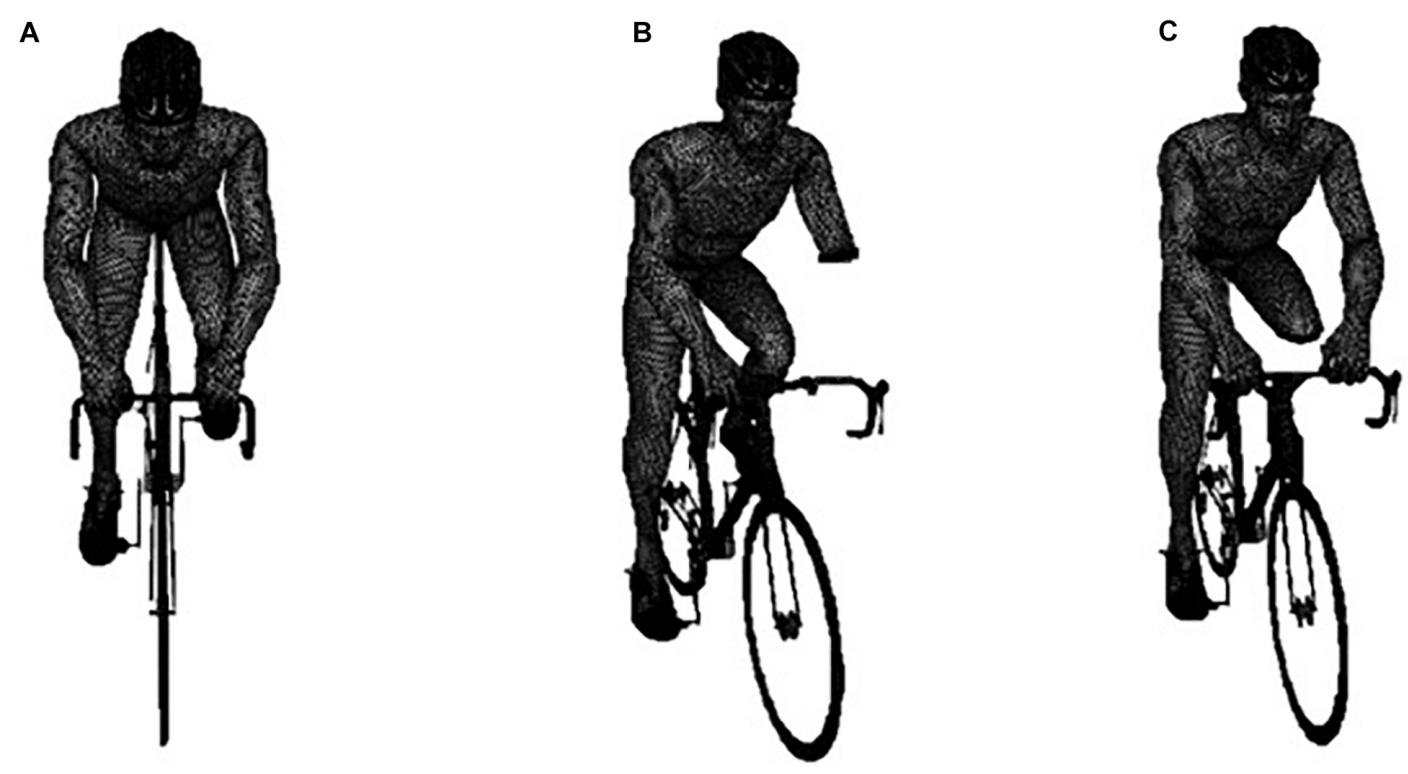

FIGURE 1 | From left to right: The meshed geometry of the able-bodied (A), transradial amputee (B), and transtibial amputee (C), on the bicycle.

tetrahedron assembly meshing; and CutCell assembly meshing. For all meshes, fine relevance center was used. The CutCell method generated the mesh with best quality and this method created a highly structured grid (Ingram et al., 2003). The other available methods present a higher computation time and the convergence does not occur at times. The simulations with the different meshes were run at $11.11 \mathrm{~m} / \mathrm{s}$, a velocity that elite cyclists typically reach during a race (Forte et al., 2020e).

In the inlet portion, velocities up to $13 \mathrm{~m} / \mathrm{s}$ with increments of $1 \mathrm{~m} / \mathrm{s}$ were set at the inlet portion of the enclosure in the geometry opposite direction ( $-\mathrm{z}$ direction). The turbulence intensity in numerical simulations was assumed as $1 \times 10^{-6} \%$. It was assumed that the bicycle-cyclist system had zero roughness non-slip wall and scalable wall functions were assigned.

\section{Numerical Simulations}

The Reynolds-Averaged Navier-Stokes (RANS) equations were solved in Fluent CFD code (Ansys Fluent 16.0, Ansys Inc., Canonsburg, PA, United States) by the finite volume approach with the Realizable k-epsilon turbulence model. The RANS model was used based on previous cycling studies (Forte et al., 2020b,d). Moreover, the Realizable k-epsilon showed higher computation economy in comparison to Standard k-epsilon, RST and RNG k-epsilon models (Defraeye et al., 2010a,b; Forte et al., 2018a; Forte et al., 2020b).

The numerical simulations were run with 3D double-precision settings and non-equilibrium wall function. For pressure-velocity coupling the SIMPLE algorithm was used. The discretization schemes were defined as second for the pressure interpolation and the convection and viscous terms. The gradients were computed by the least-squares cell-based method. Pressure and momentum were defined as second order and second order upwind. The turbulent kinetic energy and dissipation rate were defined as first order upwind. The residuals convergence criteria of the flow parameters were set to $10 \times 10^{-6}$. The residuals of the flow velocity components in the $\mathrm{x}-, \mathrm{y}-$ and $\mathrm{z}$-directions were analyzed during the simulations. The convergence occurred automatically by the Ansys Fluent 16.0 before 1,404 interactions, being the residual and drag values close to constant with negligible fluctuations.

\section{Outcomes}

\section{Drag Force}

The CFD simulations yield the total, pressure and viscous drag force, as well as, its coefficients of drag. The drag force is given by Eq. 1:

$$
F_{D}=0.5 \rho A C_{d} v^{2}
$$

$F_{D}$ is the drag force, $C_{d}$ represents the drag coefficient, $v$ the velocity, $A$ the surface area, and $\rho$ is the air density $\left(1.292 \mathrm{~kg} / \mathrm{m}^{3}\right)$. $A C_{d}$ is known as the effective surface area.

\section{Rolling Resistance}

The RR was computed by Eq. 2 .

$$
R R=C_{R} \cdot m \cdot g
$$

In Eq. $2, C_{R}$ is the rolling coefficient, $m$ the body mass of the bicycle-cyclist system, and $g$ the gravitational acceleration. The $C_{R}$ was assumed as 0.0046 on car track asphalt surface (Pugh, 1974; Candau et al., 1999; Forte et al., 2015).

The body mass was estimated based on body segment parameter (Zatsiorsky and Seluyanov, 1983; de Leva, 1996; Adolphe et al., 2017). Thus, the cyclist with $\operatorname{Tr}$ amputation had a body mass of $64.28 \mathrm{~kg}$ and the cyclist with a $\mathrm{Tt}$ amputation $63.15 \mathrm{~kg}$. 


\section{RESULTS}

The $A C_{d}$ in the able-bodied cyclist decreased with speed from 0.59 to $0.38 \mathrm{~m}^{2}$ (Figure 2). The $\mathrm{Tr}$ model $A C_{d}$ ranged between 0.58 and $0.43 \mathrm{~m}^{2}$; whereas, the Tt from 0.60 to $0.40 \mathrm{~m}^{2}$. Hence, the able-bodied model presented an $A C_{d}$ smaller than both amputees. Between 1 and $6 \mathrm{~m} / \mathrm{s}$, the $\operatorname{Tr}$ cyclist showed a smaller $A C_{d}$ in comparison to the Tt. However, at faster velocities (from 7 to $13 \mathrm{~m} / \mathrm{s}$ ) the Tt was under less drag in comparison to the Tr counterpart.

The drag ranged between 0.36 and $39.25 \mathrm{~N}$ in the able-bodied model, 0.36 and 43.78 in the Tr model, and 0.37 and $41.39 \mathrm{~N}$ in the Tt model (Figure 3). Therefore, the able-bodied cyclist was under less drag than the other two cyclists. Comparing both amputees, at slower velocities $(1-6 \mathrm{~m} / \mathrm{s})$ the $\mathrm{Tt}$ model was under more drag than $\mathrm{Tr}$. Conversely, at faster speeds $(7-12 \mathrm{~m} / \mathrm{s})$ the $\mathrm{Tr}$ model was submitted to more than $\mathrm{Tt}$.

The pressure drag ranged between 0.20 and $22.94 \mathrm{~N}$ in the able-bodied model, 0.21 and 28.61 in the Tr model, and 0.23 and 28.02 $\mathrm{N}$ in the Tt model (Figure 4). As such, one can conclude that the able-bodied cyclist was under less pressure drag than the two amputees. Again, at slow velocities (from 1 to $6 \mathrm{~m} / \mathrm{s}$ ) the $\mathrm{Tt}$ was under a larger drag force in comparison to the $\mathrm{Tr}$ counterpart. As abovementioned, at high speeds $(7-12 \mathrm{~m} / \mathrm{s})$ the Tr cyclist presented higher drag than Tt.

The viscous drag ranged between 0.16 and $15.31 \mathrm{~N}$ in the able-bodied model, 0.15 and 15.17 in the $\operatorname{Tr}$ model, and 0.14 and $13.38 \mathrm{~N}$ in the $\mathrm{Tt}$ model (Figure 5). The able-bodied model showed the largest viscous drag, followed-up by the $\mathrm{Tr}$ and $\mathrm{Tt}$ model, respectively.
The viscous and pressure drag contribution to total drag for able-bodied, Tr and Tt models are depicted in Figure 6. The ablebodied viscous drag contribution to total drag ranged between 39 and $41 \%$ and decreased with velocity. The pressure drag contribution ranged from 59 to $61 \%$ and increased with velocity. The $\mathrm{Tr}$ viscous drag ranged between 35 and 43\%, decreasing with velocity; whereas, pressure drag increased with velocity from 57 to $65 \%$. On the $\mathrm{Tt}$, viscous drag varied from 32 to $38 \%$, decreasing with velocity. The $\mathrm{Tt}$ pressure drag increased with speed between 62 and $68 \%$.

Figure 7 presents the pressure maps for the able-bodied, $\mathrm{Tr}$ and Tt. The able-bodied model presented the high pressure $8.27 \times 10^{1} \mathrm{~Pa}$, followed by the Tt with $8.49 \times 10^{1} \mathrm{~Pa}$ and the Tr with $8.74 \times 10^{1} \mathrm{~Pa}$. Moreover, the lowest pressure zones were noted on the able-bodied $\left(-3.00 \times 10^{2} \mathrm{~Pa}\right)$, followed-up by the $\mathrm{Tt}\left(-2.63 \times 10^{2} \mathrm{~Pa}\right)$ and $\operatorname{Tr}\left(-2.62 \times 10^{2} \mathrm{~Pa}\right)$.

Rolling resistance was $2.23 \mathrm{~N}$ in the able-bodied, $2.20 \mathrm{~N}$ in the $\mathrm{Tr}$, and 2.17 in the Tt (Figure 8). The difference between ablebodied and $\mathrm{Tr}$ was $1 \%$. Between the able-bodied and the $\mathrm{Tt}$ was $3 \%$, and between $\mathrm{Tr}$ and $\mathrm{Tt}$ was $2 \%$. The able-bodied presented the largest RR followed-up by the $\mathrm{Tr}$ and $\mathrm{Tt}$ model, respectively. In the three models, it is possible to note that drag represents more than $50 \%$ of the resistance at speeds over $3 \mathrm{~m} / \mathrm{s}$.

\section{DISCUSSION}

The aim of this study was to assess the aerodynamics of ablebodied and amputee cyclists. It was hypothesized that drag is higher in able-bodied cyclist, followed-up by the $\mathrm{Tr}$ and $\mathrm{Tt}$

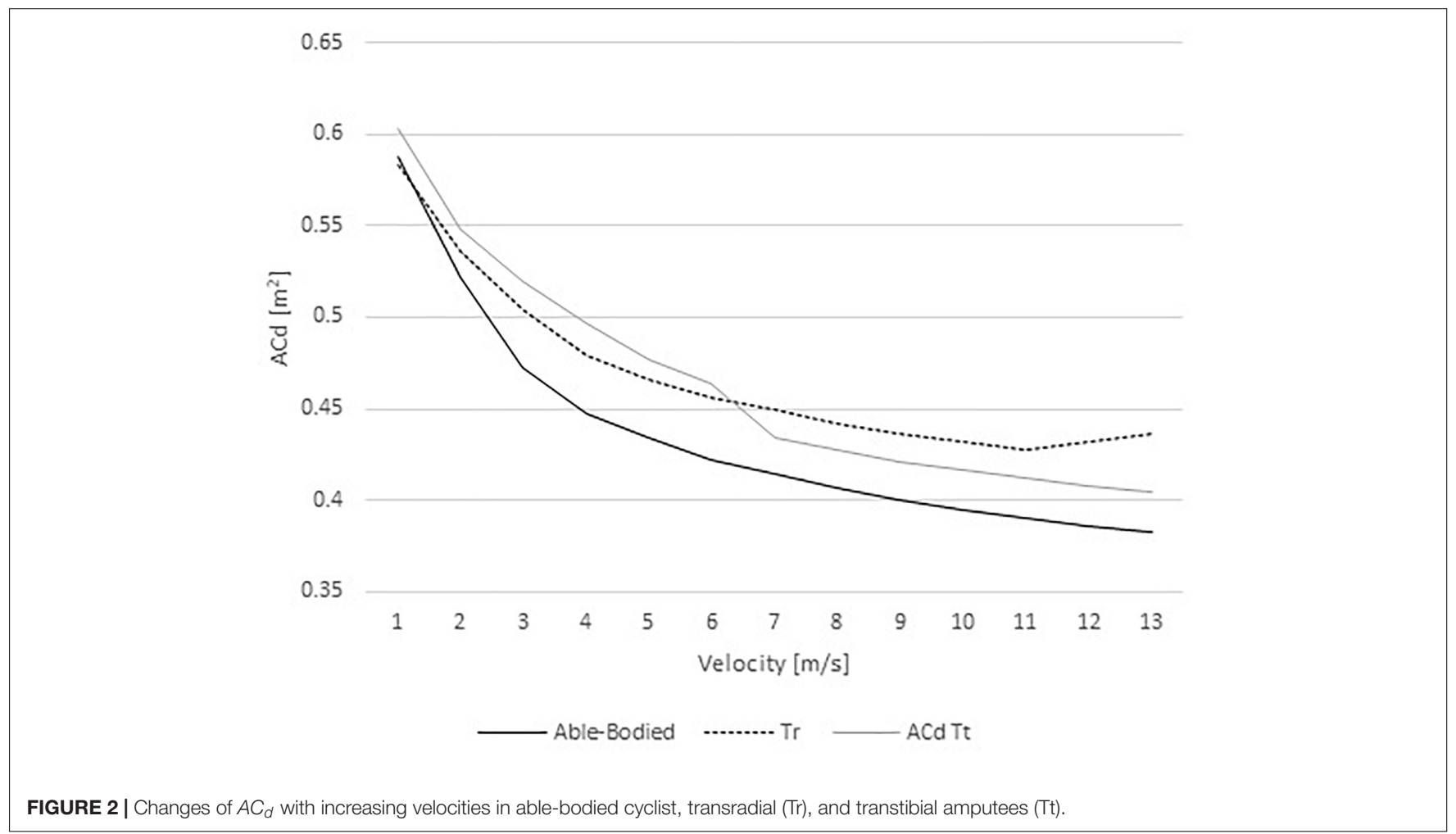




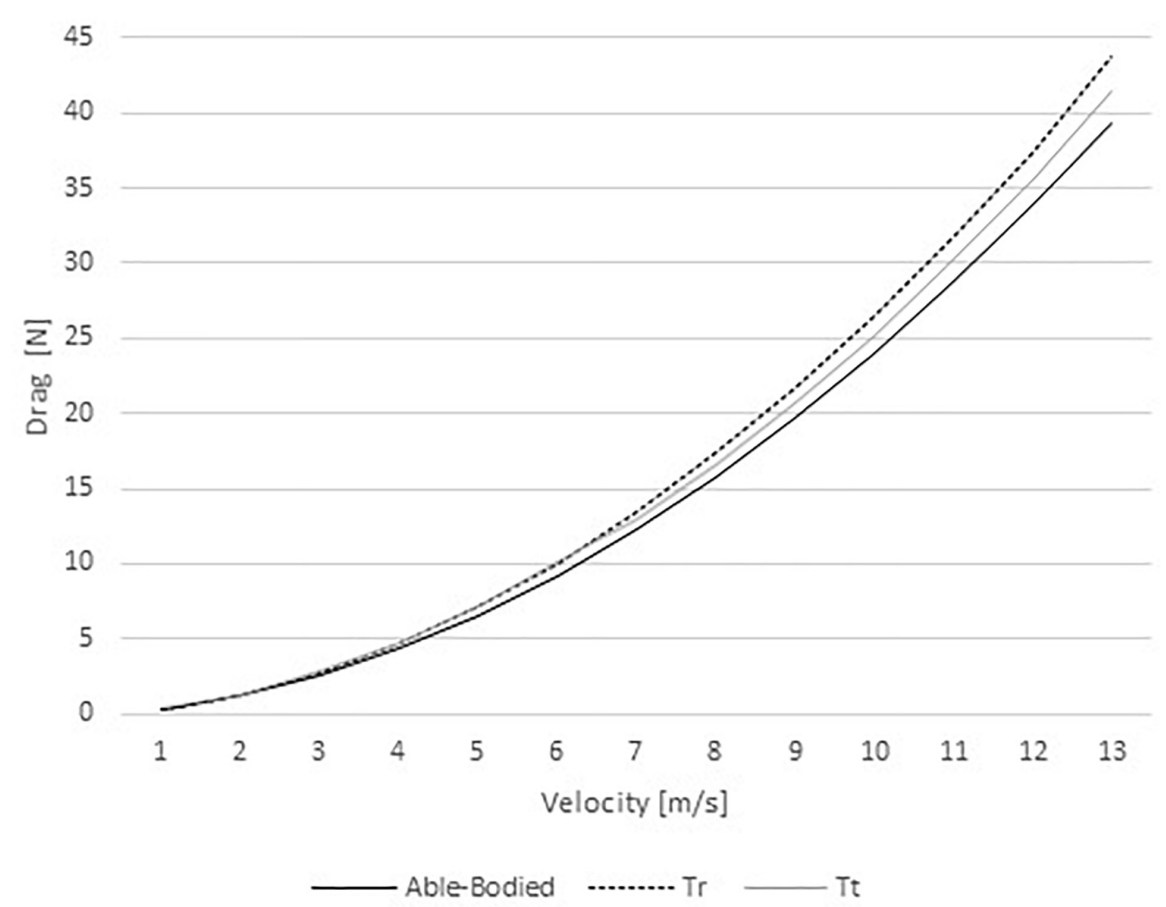

FIGURE 3 | Drag variations for able-bodied, transradial (Tr), and transtibial amputees (Tt) at the selected velocities.

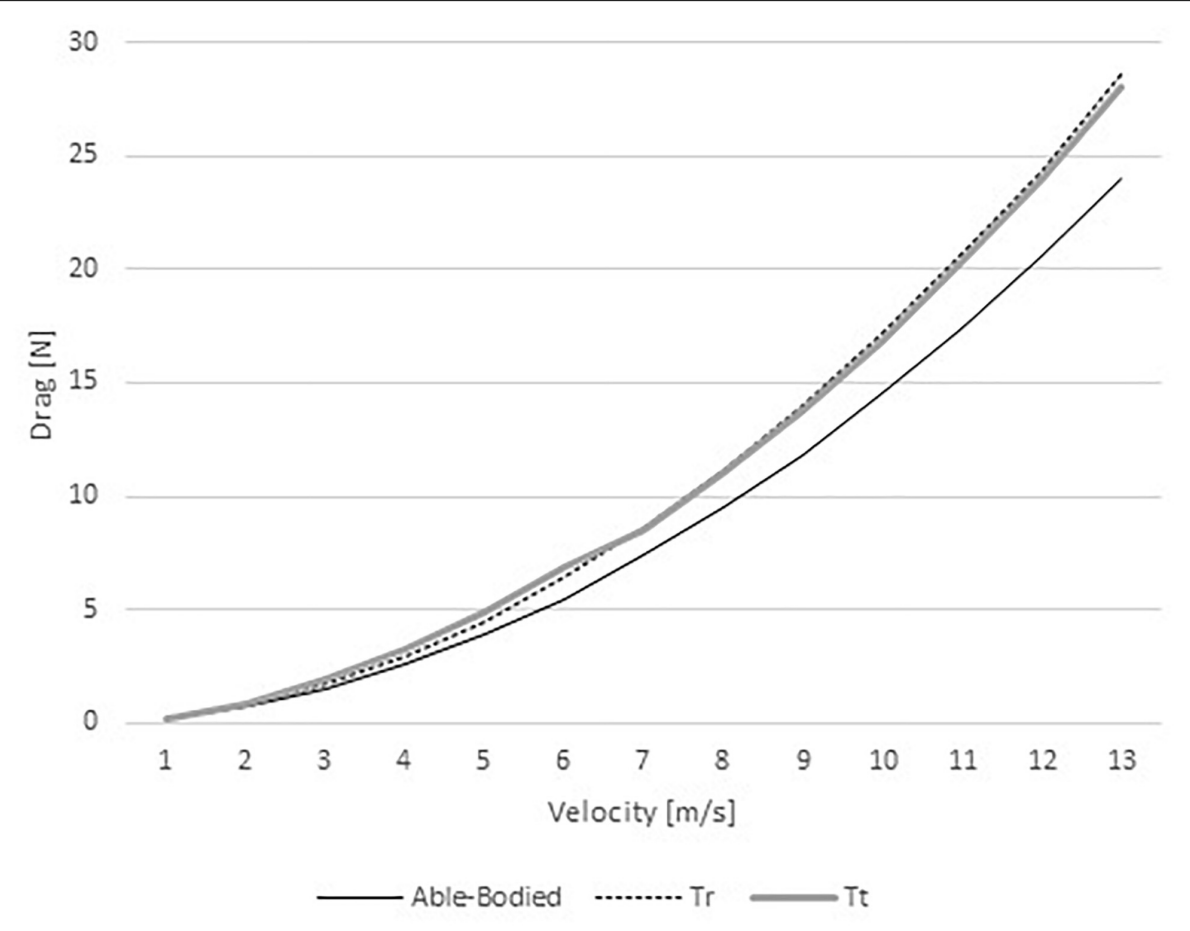

FIGURE 4 | Pressure drag variation for able-bodied, transradial (Tr), and transtibial amputees (Tt) at the selected velocities.

amputees, respectively. However, the hypothesis was rejected. The main findings of this study were that the model $\mathrm{Tr}$ was under the largest drag force in comparison to the $\mathrm{Tt}$ model and able-bodied at faster speeds (i.e., over $6 \mathrm{~m} / \mathrm{s}$ ). The ablebodied model showed the lowest drag force, in comparison to the selected amputee models. 


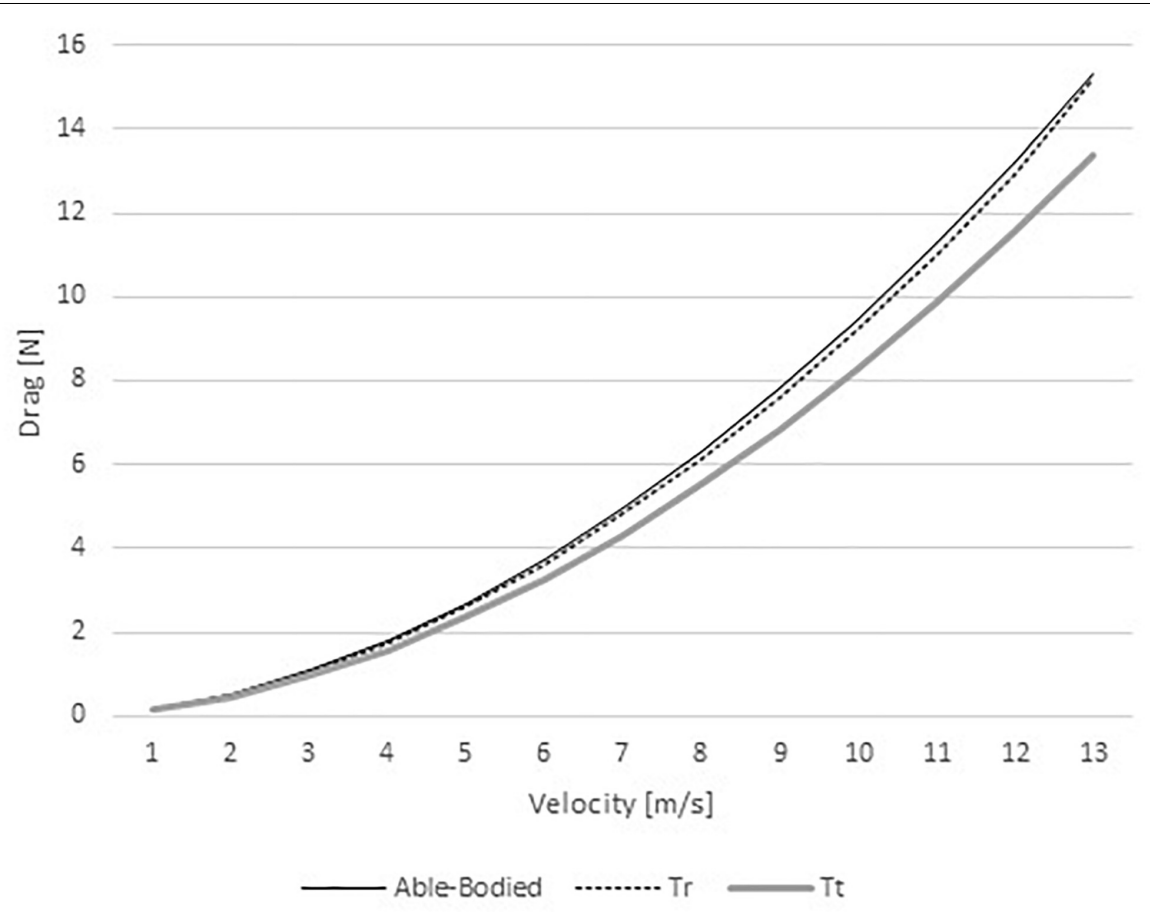

FIGURE 5 | Viscous drag variation for able-bodied, transradial (Tr), and transtibial amputees (Tt) at the selected velocities.

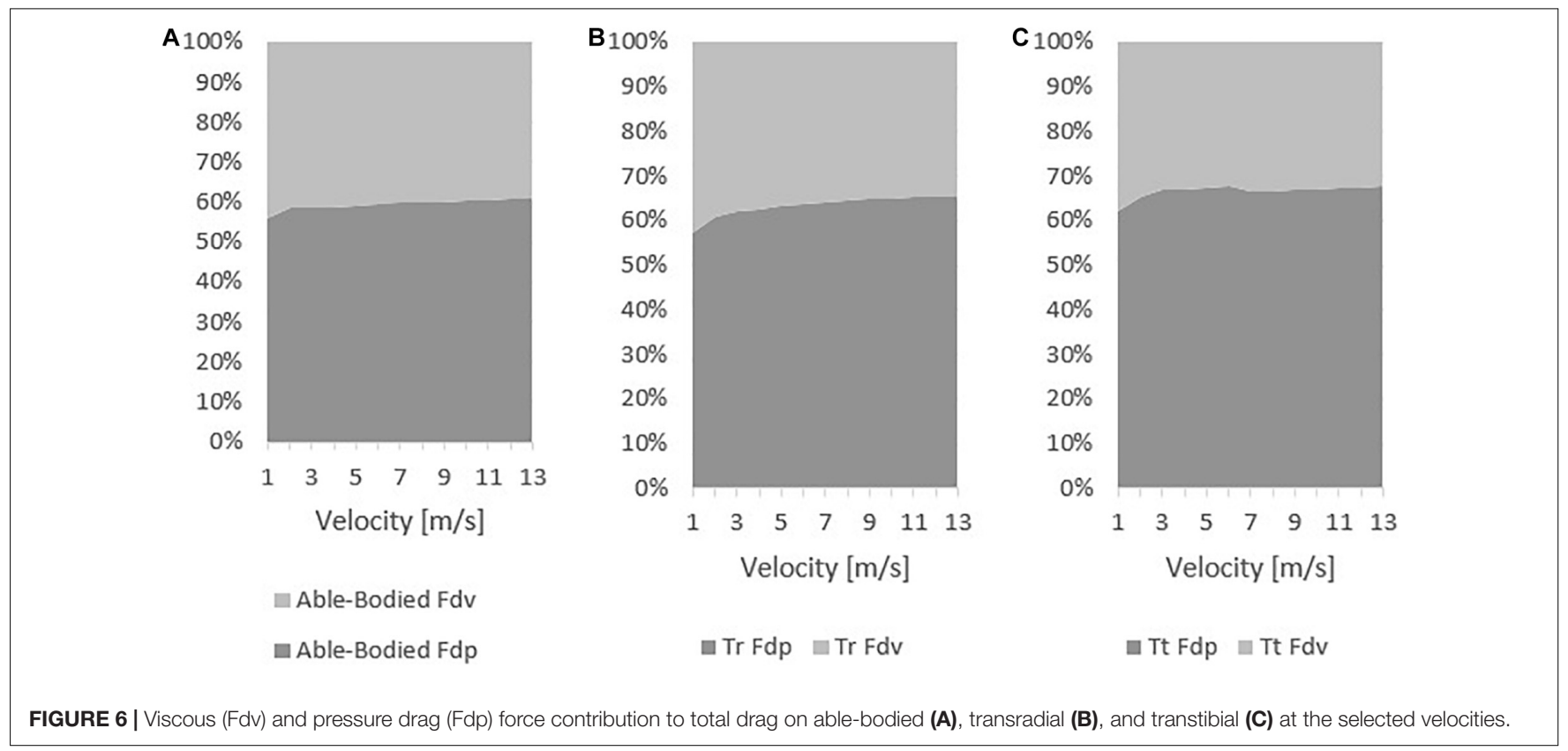

In the present study, the drag was assessed by CFD. CFD was reported as valid and reliable in comparison to wind tunnel, with differences between 7 and 11\% (Defraeye et al., 2010b). This methodology yields outputs on total drag, pressure and viscous drag components and its coefficients (Forte et al., 2018a, Forte et al., 2020a,b,c). It is also possible to estimate the energy cost of transportation of able-bodied and amputated cyclists from numerical simulations outputs (Forte et al., 2020c). The CFD data is underestimated by $18 \%$ in comparison to experimental techniques. The RR was assessed by an analytical procedures, having estimated the body mass based on body segment parameter (Zatsiorsky and Seluyanov, 1983; de Leva, 1996; Adolphe et al., 2017) and RR coefficient assumed to be 0.0046 on car track asphalt 
A

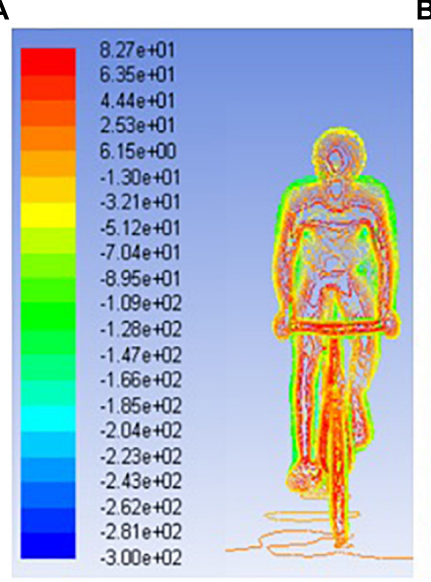

B

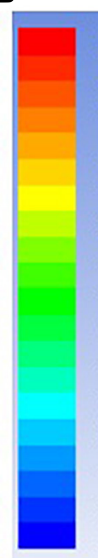

C

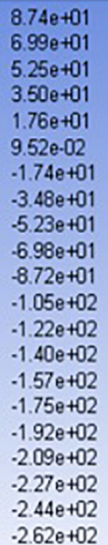

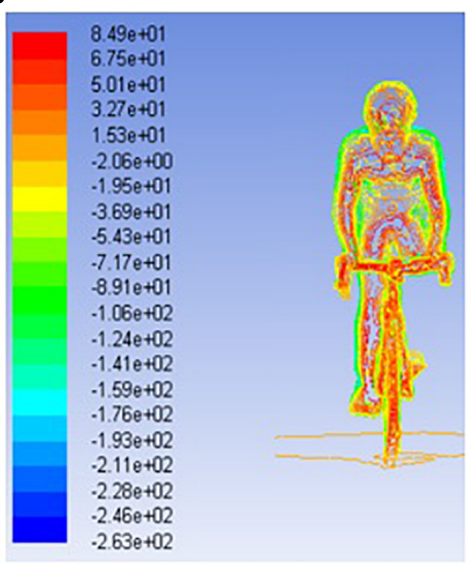

FIGURE 7 | Pressure maps for able for able-bodied (A), transradial (B), and transtibial (C) at $11 \mathrm{~m} / \mathrm{s}$.
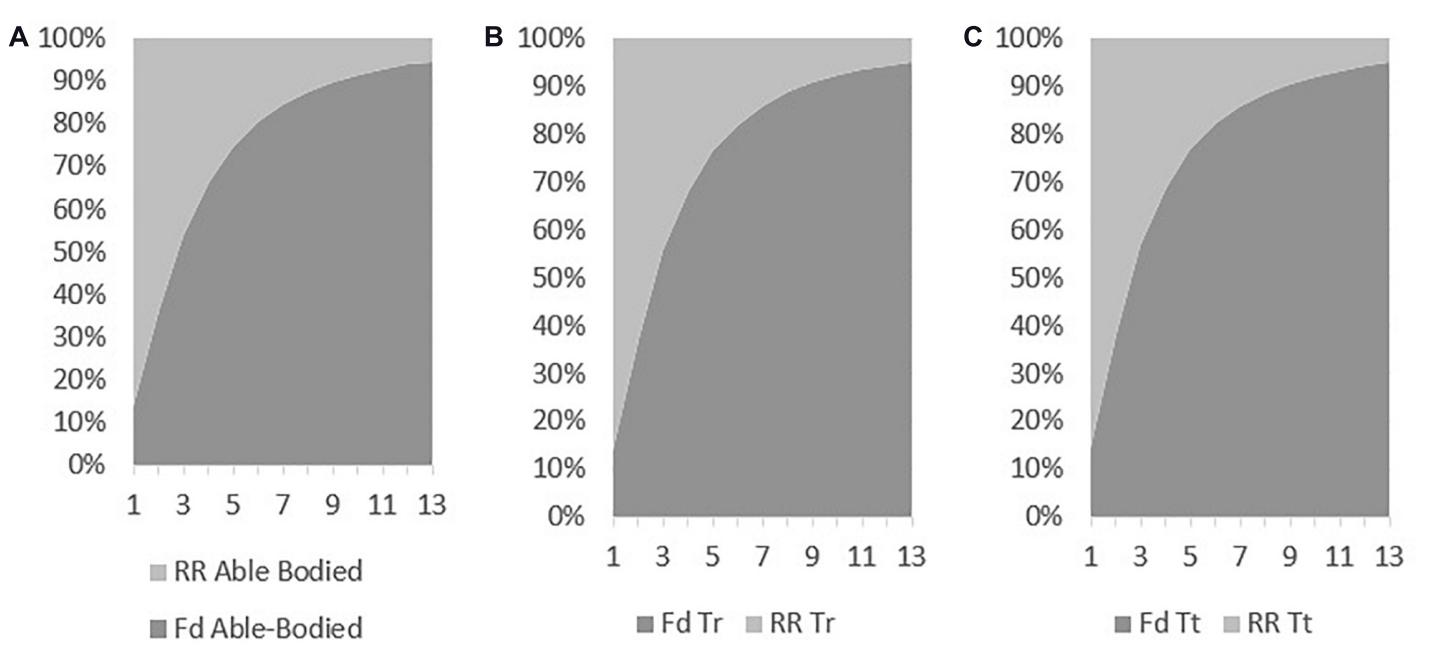

FIGURE 8 | Drag and rolling resistance contribution for total resistance for able-bodied (A), transradial (B), and transtibial (C) at the selected velocities.

surface as reported by others (Pugh, 1974; Candau et al., 1999; Forte et al., 2015).

The $A C_{d}$ in the able-bodied cyclist decreased from 0.59 to $0.38 \mathrm{~m}^{2}$ with increasing velocity. The $\operatorname{Tr}$ model $A C_{d}$ varied between 0.58 and $0.43 \mathrm{~m}^{2}$; whereas, the Tt from 0.60 to $0.40 \mathrm{~m}^{2}$. $A C_{d}$ values are in accordance to literature for velocities faster than $5 \mathrm{~m} / \mathrm{s}\left(0.37 \mathrm{~m}^{2} \leq A C_{d} \leq 0.42 \mathrm{~m}^{2}\right)$ for able-bodied cyclists (Grappe et al., 1997; Candau et al., 1999; Defraeye et al., 2010b). In other studies, the $A C_{d}$ ranged between 0.261 and $0.332 \mathrm{~m}^{2}$ for able-bodied cyclists in the upright, dropped and time-trial position (Zdravkovic et al., 1996; Grappe et al., 1997; Candau et al., 1999; Defraeye et al., 2010a; Beaumont et al., 2018; Forte et al., 2020d). The $A C_{d}$ variations are affected by $C d$ fluctuations at different speeds (Schlichting and Gersten, 2016; Forte et al., $2020 \mathrm{~d})$. It is expected that $\mathrm{Cd}$ varies about $30 \%$ on an able-bodied cyclist between 1 and $22 \mathrm{~m} / \mathrm{s}$ (Forte et al., 2020d). That can be explained by the drag crisis phenomenon. The drag crisis consists in a drop of $\mathrm{Cd}$ values due the fluid flow transition from laminar to turbulent (Schlichting and Gersten, 2016; Forte et al., 2020d). Typically, in cycling this phenomenon may occur at Re between $3.21 \times 10^{5}$ and $9.63 \times 10^{5}$ and, speeds between 3 and $9 \mathrm{~m} / \mathrm{s}$. In cycling the drag crisis was assessed in the time trial position (Forte et al., 2020d). However, the present analysis was carried out in the upright position. Moreover, drag coefficient variations can be explained by the geometry shape/form, dimensions and the changes in the upright position might be different from the time trial position (Schlichting and Gersten, 2016; Forte et al., $2020 \mathrm{~d})$. The $A C_{d}$ values also explain the drag variations across different speeds. Drag is dependent on the air density, velocity, surface area, and drag coefficient. The drag values were smaller on the able-bodied model, Tr was under more drag than Tt model, at faster speeds (i.e., over $6 \mathrm{~m} / \mathrm{s}$ ). However, Forte et al. (2020a), reported drag values between 19.66 and $21.98 \mathrm{~N}$ for an ablebodied model in the dropped position. These values are slightly below the results of the present study at the same velocity (28.67$31.71 \mathrm{~N})$. Forte et al. (2020a), noted that cyclists in the dropped 
position have a smaller effective surface area (dropped position: 0.30 and $0.41 \mathrm{~m}^{2}$; upright position: 0.37 to $0.42 \mathrm{~m}^{2}$ ). Blocken et al. (2018a) reported an $A C_{d}$ of $0.277 \mathrm{~m}^{2}$ in the dropped position matching $20.90 \mathrm{~N}$ in the same conditions of our study. This study was conducted in static position and the literature reported a good agreement between steady and unsteady analyses in cycling (Crouch et al., 2014; Griffith et al., 2019). Notwithstanding, amputation can cause body and bicycle rotations (Koutny et al., 2013). At least, in human swimming, this body rotation due to amputations was already assessed by CFD (Lecrivain et al., 2010).

Pressure drag values were lower for the able-bodied model, followed-up by the Tr and Tt models, respectively. Pressure drag is due to pressure differences between the front and back boundaries (Schlichting and Gersten, 2016). The fluid separation from the object at the back-boundary results in a low pressure zone due the object form/shape (Debraux et al., 2011; Schlichting and Gersten, 2016; Forte et al., 2020d). At least one study reported the pressure drag of a cyclist as being $12.56-16.51 \mathrm{~N}$ at $11.11 \mathrm{~m} / \mathrm{s}$, depending on the gear the subject was wearing (in the case the type of helmet). The pressure drag contribution to total drag was between 63 and 75\% (Forte et al., 2020a). Other study but of an elite wheelchair sprinter in Athletics noted a pressure drag of $5.5 \mathrm{~N}$ at $6.5 \mathrm{~m} / \mathrm{s}$, accounting to $64 \%$ of total drag. In the present study at $7 \mathrm{~m} / \mathrm{s}$ was $7 \mathrm{~N}$, and at $11 \mathrm{~m} / \mathrm{s}$ near $15-22 \mathrm{~N}$. The contribution of pressure drag in the present study ranged between 58 and $68 \%$. Therefore, the differences between the present study and literature can be explained by the position under assessment (Forte et al., 2020a), and differences in sport events (wheelchair racing vs. cycling) at different speeds (Forte et al., 2018a). In the present study, the pressure maps depicted less pressure in the able-bodied, followed-up by the $\mathrm{Tt}$ and then the Tr. That can be explained by the differences of pressure between back and front boundaries, added that the fluid flow turbulence may increase due the object shape (Schlichting and Gersten, 2016; Forte et al., 2020d). Moreover, the $\mathrm{Tr}$ and $\mathrm{Tt}$ amputations may affect the fluid flow turbulence, generating vorticities that ultimately are going to influence the drag (Schlichting and Gersten, 2016). This can explain the larger pressure drag acting on $\mathrm{Tr}$ and Tt. No study was founded sharing pressure maps in amputee cyclists. Thus, it is challenging to benchmark our finding with others. The order from highest to lowest pressure and pressure drag was the same. That may also justify the differences in pressure drag. The highest was the $\mathrm{Tr}$, then the $\mathrm{Tt}$ and finally the able-bodied. This can explain why able-bodied cyclist was under less drag than the other counterparts under analysis. The amputations lead to more fluid turbulence around the limbs, the body, the cyclist-bicycle system and hence, to larger pressure differences (Forte et al., 2018a; Forte et al., 2020a).

The viscous drag ranged between 0.16 and $15.31 \mathrm{~N}$ in the able-bodied cyclist, 0.15 and $15.17 \mathrm{~N}$ in the Tr model, and 0.14 and $13.38 \mathrm{~N}$ in the $\mathrm{Tt}$ model. Once more, it was not possible to find studies on cyclist's viscous drag in the literature. Viscous drag is related to the amount of layers of fluid that are dragged by the body (Schlichting and Gersten, 2016; Forte et al., 2018a). Larger bodies (i.e., bodies with larger surfaces) are prone to be under more viscous drag (Schlichting and Gersten, 2016; Forte et al., 2018a). Reductions in surface area, for instance, adopting different body postures, can decrease viscous drag (Forte et al., 2018a). At least one study reported that viscous drag was 10.52 and $16.51 \mathrm{~N}$ at $11.11 \mathrm{~m} / \mathrm{s}$ in cyclists wearing aero and normal road helmet, respectively (Forte et al., 2020a). As expected, the able-bodied model of the present study was under larger viscous drag because it was the one with largest surface area. The amputee models presented less surface area, explaining the differences in viscous drag (Schlichting and Gersten, 2016; Forte et al., 2018a). The viscous drag contribution to total drag varied between 32 and $42 \%$ and decreased with velocity in the different models. At slower velocities, viscous drag is the main resistive force and the result of the system's shape/form (Forte et al., 2015; Schlichting and Gersten, 2016).

Drag is the cyclist's main resistive force at velocities over $5 \mathrm{~m} / \mathrm{s}$ (Martin et al., 2006). In our study for velocities over $5 \mathrm{~m} / \mathrm{s}$ the total drag was the highest in the Tr model, then in the Tt and lastly in the able-bodied models. The drag analyses were made in static positions. At least one study reported that the differences between static positions and dynamic leg-motions affect $\mathrm{Cd}$ in 6\% (Crouch et al., 2016). Thus, under ecological conditions (i.e., pedaling), the drag might be $6 \%$ larger than our results. However, it is not possible to design an experimental or quasi-experimental cross-over design to compare the aerodynamics of an able-bodied and amputee. CFD remains as the most controlled technique to gather insights on this matter. Moreover, the majority of the studies that assessed cyclists aerodynamics are in static positions (Defraeye et al., 2010b; Blocken et al., 2013, Blocken and Toparlar, 2015; Blocken et al., 2018b; Beaumont et al., 2018). The total drag ranged between 0.36 and $43.78 \mathrm{~N}$ across the different models and speeds. The literature presents cyclists drag values between 0.16 and $76.45 \mathrm{~N}$ at different positions (upright, dropped and time trial) and speeds (from 1 to $22 \mathrm{~m} / \mathrm{s}$ ) (Forte et al., 2020a,d). It is possible to find $A C_{d}$ values between 0.261 and $0.42 \mathrm{~m}^{2}$ (Zdravkovic et al., 1996; Grappe et al., 1997; Candau et al., 1999; Defraeye et al., 2010a; Beaumont et al., 2018; Forte et al., 2020d). Considering this study settings, for these $A C_{d}$ values, the drag may vary between 0.16 and $43.40 \mathrm{~N}$. Altogether, the present study shows a good agreement with literature.

The pressure drag was also the highest in the Tr model, followed-up by the Tt and able-bodied model. Conversely, the viscous drag was the highest in the able-bodied model, then the Tr model and last the Tt model. The pressure drag contribution ranged between 58 and 68\%. The able-bodied model presented the high pressure, followed by the $\mathrm{Tt}$ and the Tr. The pressure zones highest values were $8.27 \times 10^{1}$, $8.49 \times 10^{1}$, and $8.74 \times 10^{1} \mathrm{~Pa}$ for the able-bodied, Tt, and $\mathrm{Tr}$, respectively. We failed to find studies assessing the pressure maps (contours) of bicycle-cyclist system. Most of the studies assessed pressure coefficients and relative velocity magnitude. The pressure differences are mainly caused by the pressure differences between the system's front and back boundaries (Forte et al., 2015; Schlichting and Gersten, 2016). The pressure differences can possibly be explained by the vorticity around the amputee's limbs. However, there is a lack of studies assessing the aerodynamics of amputees. More research is needed to better understand this phenomenon. At faster velocities, pressure drag had the highest contribution to total drag (Forte et al., 2020a). 
The $\mathrm{RR}$ was $2.23 \mathrm{~N}$ in the able-bodied, $2.20 \mathrm{~N}$ in the $\mathrm{Tr}$ and 2.17 in the Tt. RR is dependent on the bicycle-cyclists mass and the rolling friction coefficient (Forte et al., 2018b). The RR coefficient was assumed to be 0.0046 on car track asphalt surface as reported in the literature (Candau et al., 1999; Forte et al., 2018 b). In our study, the able-bodied model was the one with more mass of the three. Then, it was the $\mathrm{Tr}$ and finally the $\mathrm{Tt}$ models. Hence, it was expected RR to be larger on the ablebodied model. The RR contribution to total resistance force ranged between 10 and $90 \%$. The $\mathrm{RR}$ contributions decreased with increasing velocity; whereas, drag contribution increased with the velocity. Thus, there is a trade-off in the contributions of between RR and total drag to total resistance force with increasing velocity. Drag force represented more than $80 \%$ of the total resistance force at $5 \mathrm{~m} / \mathrm{s}$. At the same velocity, it is expected that drag may contribute about $90 \%$ of the resistive forces (Martin et al., 2006). This value is within the results of our study with different models. Moreover, in an elite wheelchair sprinter, at the world record speed, RR was about 40\% (Forte et al., 2018b). However, wheelchair sprinters in Athletics have larger surface areas and their maximal speed is slower than cyclists (Blocken et al., 2018a; Forte et al., 2020e).

This study reported for the first time the aerodynamics of two different amputee and compared it to an able-bodied cyclist. The main findings were that viscous, pressure and total drag vary depending on the type of amputation. Viscous drag and pressure drag are mainly affected by the amputation and the latter has an influence on the fluid flow around the limbs, body and bicyclecyclist system. Altogether, at mean velocity, drag was higher in Tr, followed-up by $\mathrm{Tt}$ and then the able-bodied model. Viscous drag was the highest in the able-bodied due the larger surface area. The $\mathrm{Tr}$ and $\mathrm{Tt}$ amputation diminish the surface area and, thus the viscous drag. The pressure drag was the highest in the Tr, then the $\mathrm{Tt}$ and lastly the able-bodied model. That can be explained by the fluid distortions and differences of pressure between the back and front boundaries in the $\mathrm{Tr}$ and Tt models. RR was higher in able-bodied, followed-up by the Tr and then the Tt. Differences in RR are explained by the mass differences; where, able-bodied had more mass, followed by the $\mathrm{Tr}$ and $\mathrm{Tt}$ the lightest of the three. Findings from this study can also aid cycling committees to set specific para-sport rules.

This study has the following limitations: (1) only one ablebodied cyclist was recruited; (2) this study did not assess how the use of prosthesis can affect the aerodynamics; (3) different

\section{REFERENCES}

Adolphe, M., Clerval, J., Kirchof, Z., Lacombe-Delpech, R., and Zagrodny, B. (2017). Center of mass of human's body segments. Mech. Mech. Eng. 21, 485-497.

Beaumont, F., Taiar, R., Polidori, G., Trenchard, H., and Grappe, F. (2018). Aerodynamic study of time-trial helmets in cycling racing using CFD analysis. J. Biomech. 67, 1-8. doi: 10.1016/j.jbiomech.2017. 10.042

Blocken, B., Defraeye, T., Koninckx, E., Carmeliet, J., and Hespel, P. (2013). CFD simulations of the aerodynamic drag of two drafting cyclists. Comput. Fluids. 71, 435-445. doi: 10.1016/j.compfluid.2012.11.012 environmental conditions such as weather conditions (e.g., air temperature) were not assessed; (4) steady analyses were run even though the good agreement with unsteady assessments.

\section{CONCLUSION}

The able-bodied cyclist model was the one under less drag force, followed-up by the Tt model and then the Tr model. At faster velocities (i.e., over $6 \mathrm{~m} / \mathrm{s}$ ), pressure drag was larger in the $\mathrm{Tr}$ cyclist, followed-up by the $\mathrm{Tt}$ and the able-bodied counterparts, respectively. In the case of the viscous drag, the able-bodied model showed the highest values, then the $\operatorname{Tr}$ and finally the Tt. The RR was higher in the case of the able-bodied, being second the Tr model and third the Tt model. In summary, the aerodynamics varies according to cyclists' classification.

\section{DATA AVAILABILITY STATEMENT}

The raw data supporting the conclusions of this article will be made available by the authors, without undue reservation.

\section{ETHICS STATEMENT}

The Scientific Committee of the Douro Higher Institute of Educational Sciences approved this research. The patients/participants provided their written informed consent to participate in this study.

\section{AUTHOR CONTRIBUTIONS}

PF, JM, and DM conceived and designed the experiments. JM and $\mathrm{PF}$ performed the experiments. PF and TB analyzed the data. PF and JM drafted the manuscript. TB and DM revised the manuscript. All authors contributed to the article and approved the submitted version.

\section{FUNDING}

This research was supported by the Portuguese Foundation for Science and Technology, I.P. (project UIDB04045/2020).

Blocken, B., and Toparlar, Y. (2015). A following car influences cyclist drag: CFD simulations and wind tunnel measurements. J. Wind Eng. Ind. Aerodyn. 145, 178-186. doi: 10.1016/j.jweia.2015.06.015

Blocken, B., van Druenen, T., Toparlar, Y., and Andrianne, T. (2018a). Aerodynamic analysis of different cyclist hill descent positions. J. Wind. Eng. Ind. Aerodyn. 181, 27-45. doi: 10.1016/j.jweia.2018.08.010

Blocken, B., van Druenen, T., Toparlar, Y., Malizia, F., Mannion, P., Andrianne, T., et al. (2018b). Aerodynamic drag in cycling pelotons: new insights by CFD simulation and wind tunnel testing. J. Wind Eng. Ind. Aerodyn. 179, 319-337. doi: 10.1016/j.jweia.2018.06.011

Candau, R. B., Grappe, F., Menard, M., Barbier, B., Millet, G. Y., Hoffman, M. D., et al. (1999). Simplified deceleration method for assessment of resistive forces 
in cycling. Med. Sci. Sports Exerc. 31, 1441-1447. doi: 10.1097/00005768199910000-00013

Childers, W., Gallagher, T., Duncan, J., and Taylor, D. K. (2015). Modeling the effect of a prosthetic limb on $4-\mathrm{km}$ pursuit performance. Int. J. Sports Physiol. Perform. 10, 3-10. doi: 10.1123/ijspp.2013-0519

Crouch, T. N., Burton, D., Thompson, M., Martin, D. T., Brown, N. A. T., and Sheridan, J. (2014). "A phase-averaged analysis of the pedalling cyclist wake," in Proceedings of the 19th Australian Fluid Mechanics Conference, Melbourne, VIC, 100-115.

Crouch, T. N., Burton, D., Thompson, M. C., Brown, N. A. T., and Sheridan, J. (2016). Dynamic leg-motion and its effect on the aerodynamic performance of cyclists. J. Fluids Struct. 65, 121-137. doi: 10.1016/j.jfluidstructs.2016. 05.007

de Leva, P. (1996). Adjustments to Zatsiorsky-Seluyanov's segment in ertia parameters. J. Biomech. 29, 1223-1230. doi: 10.1016/0021-9290(95) 00178-6

Debraux, P., Grappe, F., Manolova, A. V., and Bertucci, W. (2011). Aerodynamic drag in cycling: methods of assessment. Sports Biomech. 10, 197-218. doi: 10. 1080/14763141.2011.592209

Defraeye, T., Blocken, B., Koninckx, E., Hespel, P., and Carmeliet, J. (2010a). Aerodynamic study of different cyclist positions: CFD analysis and full-scale wind-tunnel tests. J. Biomech. 43, 1262-1268. doi: 10.1016/j.jbiomech.2010. 01.025

Defraeye, T., Blocken, B., Koninckx, E., Hespel, P., and Carmeliet, J. (2010b). Computational fluid dynamics analysis of cyclist aerodynamics: performance of different turbulence-modelling and boundary-layer modelling approaches. J. Biomech. 43, 2281-2287. doi: 10.1016/j.jbiomech.2010. 04.038

Dyer, B. (2014). The importance of aerodynamics for prosthetic limb design used by competitive cyclists with an amputation: an introduction. Prosthet. Orthot. Int. 39, 232-237. doi: 10.1177/0309364614527121

Dyer, B. (2016). Cycling with an amputation: a systematic review. Prosthet. Orthot. Int. 40, 538-544. doi: 10.1177/0309364615610659

Dyer, B., and Disley, B. X. (2017). Validation of the virtual elevation field test method when assessing the aerodynamics of para-cyclists with a uni-lateral trans-tibial amputation. Disabil. Rehabil. Assist. Technol. 13, 107-111. doi: 10. 1080/17483107.2017.1297857

Forte, P., Barbosa, T. M., and Marinho, D. A. (2015). “Technologic appliance and performance concerns in wheelchair racing - helping Paralympic athletes to excel," in New Perspectives in Fluid Dynamics, ed. C. Liu (Rijeka: IntechOpen), 101-121.

Forte, P., Marinho, D. A., Barbosa, T. M., and Morais, J. E. (2020a). Analysis of a normal and aero helmet on an elite cyclist in the dropped position. AIMS Biophys. 7, 54-64. doi: 10.3934/biophy.2020005

Forte, P., Marinho, D. A., Morais, J. E., Morouço, P. G., and Barbosa, T. M. (2018a). Estimation of mechanical power and energy cost in elite wheelchair racing by analytical procedures and numerical simulations. Comput. Methods Biomech. Biomed. Engin. 21, 585-592. doi: 10.1080/10255842.2018.1502277

Forte, P., Marinho, D. A., Morais, J. E., Morouço, P. G., and Barbosa, T. M. (2018b). The variations on the aerodynamics of a world-ranked wheelchair sprinter in the key-moments of the stroke cycle: a numerical simulation analysis. PLoS One 13:e0193658. doi: 10.1371/journal.pone.0193658

Forte, P., Marinho, D. A., Nikolaidis, P. T., Knechtle, B., Barbosa, T. M., and Morais, J. E. (2020b). Analysis of cyclist's drag on the aero position using numerical simulations and analytical procedures: a case study. Int. J. Environ. Res. Public Health 17:3430. doi: 10.3390/ijerph17103430

Forte, P., Marinho, D. A., Silveira, R., Barbosa, T. M., and Morais, J. E. (2020c). The aerodynamics and energy cost assessment of an able-bodied cyclist and amputated models by computer fluid dynamics. Medicina 56:241. doi: 10.3390/ medicina56050241

Forte, P., Morais, J. E., Neiva, H. P., Barbosa, T. M., and Marinho, D. A. (2020d). The drag crisis phenomenon on an elite road cyclist-a preliminary numerical simulations analysis in the aero position at different speeds. Int. J. Environ. Res. Public Health 17:5003. doi: 10.3390/ijerph17145003

Forte, P., Morouço, P., Barbosa, T. M., Marinho, D., and Morais, J. (2020e). Estimation of an elite road cyclist performance in different positions based on numerical simulations and analytical procedures. Front. Bioeng. Biotech. 8:538. doi: 10.3389/fbioe.2020.00538

Grappe, F., Candau, R., Belli, A., and Rouillon, J. D. (1997). Aerodynamic drag in field cycling with special reference to the Obree's position. Ergonomics 40, 1299-1311. doi: 10.1080/001401397187388

Griffith, M. D., Crouch, T. N., Burton, D., Sheridan, J., Brown, N. A., and Thompson, M. C. (2019). A numerical model for the time-dependent wake of a pedalling cyclist. Proc. Inst. Mech. Eng. P J. Sport. Eng. Technol. 233, 514-525. doi: $10.1177 / 1754337119858434$

Ingram, D. M., Causon, D. M., and Mingham, C. G. (2003). Developments in Cartesian cut cell methods. Math. Comput. Simul. 61, 561-572. doi: 10.1016/ s0378-4754(02)00107-6

Koutny, D., Palousek, D., Stoklasek, P., Rosicky, J., Tepla, L., Prochazkova, M., et al. (2013). The biomechanics of cycling with a transtibial prosthesis: a case study of a professional cyclist. Int. J. Med. Health Biomed. Bioeng. Pharm. Eng. 7, 812-817.

Lecrivain, G., Payton, C., Slaouti, A., and Kennedy, I. (2010). Effect of body roll amplitude and arm rotation speed on propulsion of arm amputee swimmers. $J$ Biomech. 43, 1111-1117. doi: 10.1016/j.jbiomech.2009.12.014

Liljedahl, J. B., Bjerkefors, A., Arndt, A., and Nooijen, C. F. (2020). Para-cycling race performance in different sport classes. Disabil. Rehabil. 14, 1-5. doi: 10.1080/09638288.2020.1734106

Marinho, D. A., Barbosa, T. M., Reis, V. M., Kjendlie, P. L., Alves, F. B., Vilas-Boas, J. P., et al. (2010). Swimming propulsion forces are enhanced by a small finger spread. J. Appl. Biomech. 26, 87-92. doi: 10.1123/jab.26.1.87

Martin, J., Gardner, S., Barras, M., and Martin, D. T. (2006). Modelling sprint cycling using field-derived parameters and forward integration. Med. Sci. Sports Exerc. 38, 592-597. doi: 10.1249/01.mss.0000193560.34022.04

Martin, J. C., Milliken, D. L., Cobb, J. E., McFadden, K. L., and Coggan, A. R. (1998). Validation of a mathematical model for road cycling power. J. Appl. Biomech. 14, 276-291. doi: 10.1123/jab.14.3.276

Peters, M. (2009). "Computational fluid dynamics for sport simulation," Computational Science and Engineering. (Berlin: Springer), 18.

Pugh, L. (1974). The relation of oxygen intake and speed in competition cycling and comparative observations on the bicycle ergometer. J. Physiol. 241, 795-808. doi: 10.1113/jphysiol.1974.sp010685

Schlichting, H., and Gersten, K. (2016). Boundary-Layer Theory. Berlin: Springer.

Tweedy, S. M., and Vanlandewijck, Y. C. (2011). International Paralympic Committee position stand-background and scientific principles of classification in Paralympic sport. Br. J. Sports Med. 45, 259-269. doi: 10.1136/ bjsm.2009.065060

Zatsiorsky, V., and Seluyanov, V. (1983). "The mass and inertia characteristics of the main segments of the human body," in Biomechanics VIII-B, eds H. Matsui and K. Kobayashi (Champaign, IL: Human Kinetic), 1152-1159.

Zdravkovic, M. M., Ashcroft, M. W., Chisholm, S. J., and Hicks, N. (1996). Effect of cyclist's posture and vicinity of another cyclist on aerodynamic drag. The Eng. Sport. 1, 21-28.

Conflict of Interest: The authors declare that the research was conducted in the absence of any commercial or financial relationships that could be construed as a potential conflict of interest.

Copyright (C) 2021 Forte, Morais, Barbosa and Marinho. This is an open-access article distributed under the terms of the Creative Commons Attribution License (CC BY). The use, distribution or reproduction in other forums is permitted, provided the original author(s) and the copyright owner(s) are credited and that the original publication in this journal is cited, in accordance with accepted academic practice. No use, distribution or reproduction is permitted which does not comply with these terms. 